\title{
Existence of solutions for nonlinear singular fractional differential equations with fractional derivative condition
}

Rengui Li*

\section{"Correspondence:}

314234634@qq.com

Department of Mathematics, Jining

University, Jining, Shandong

273155, P.R. China

\begin{abstract}
In this paper, we investigate a boundary value problem for singular fractional differential equations with a fractional derivative condition. The existence and uniqueness of solutions are obtained by means of the fixed point theorem. Some examples are presented to illustrate our main results.
\end{abstract}

Keywords: boundary value problem; singular fractional differential equation; Caputo fractional derivative; fixed point theory

\section{Introduction}

Differential equations of fractional order have recently been addressed by many researchers of various fields of science and engineering such as physics, chemistry, biology, economics, control theory, and biophysics; see $[1,2]$. On the other hand, fractional differential equations also serve as an excellent tool for the description of memory and hereditary properties of various materials and processes. With these advantages, the model of fractional order become more and more practical and realistic than the classical of integer order, such effects in the latter are not taken into account. As a result, the subject of fractional differential equations is gaining much attention and importance.

Recently, much attention has been focused on the study of the existence and uniqueness of solutions for boundary value problem of fractional differential equations with nonlocal boundary conditions by the use of techniques of nonlinear analysis (fixed point theorems, Leray-Schauder theory, the upper and lower solution method, etc.); see [3-17].

In [18], Agarwal et al. investigated the existence of solutions for the singular fractional boundary value problems

$$
\left\{\begin{array}{l}
D^{\alpha} u(t)+f\left(t, u(t), D^{\mu} u(t)\right)=0, \quad 0<t<1, \\
u(0)=u(1)=0
\end{array}\right.
$$

where $1<\alpha<2,0<\mu \leq \alpha-1$ are real numbers, $D^{\alpha}$ is the standard Riemann-Liouville fractional derivative, $f$ satisfies the Caratheodory conditions on $[0,1] \times(0, \infty) \times R, f$ is positive, and $f(t, x, y)$ is singular at $x=0$.

๑2014 Li; licensee Springer. This is an Open Access article distributed under the terms of the Creative Commons Attribution License (http://creativecommons.org/licenses/by/2.0), which permits unrestricted use, distribution, and reproduction in any medium, provided the original work is properly cited. 
In [19], Yan et al. studied the existence and uniqueness of solutions for a class of fractional differential equations with integral boundary conditions

$$
\left\{\begin{array}{l}
{ }^{C} D_{0^{+}}^{\alpha} x(t)+f\left(t, x(t),{ }^{C} D_{0^{+}}^{\beta} x(t)\right)=0, \quad t \in[0,1] \\
x(0)+x^{\prime}(0)=y(x), \quad \int_{0}^{1} x(t) d t=m \\
x^{\prime \prime}(0)=x^{\prime \prime \prime}(0)=\cdots=x^{(n-1)}(0)=0
\end{array}\right.
$$

where ${ }^{C} D_{0^{+}}^{\alpha},{ }^{C} D_{0^{+}}^{\beta}$ are the Caputo fractional derivatives, $f:[0,1] \times R \times R \rightarrow R$ is a continuous function, $y:[0,1] \rightarrow R$ is a continuous function, and $m \in R, n-1<\alpha<n(n \geq 2)$, $0<\beta<1$ is a real number.

In [20], Guezane-Lakoud and Bensebaa discussed the existence and uniqueness of solutions for a fractional boundary value problem with a fractional derivative condition,

$$
\left\{\begin{array}{l}
{ }^{C} D_{0^{+}}^{q} u(t)+f\left(t, u(t),{ }^{C} D_{0^{+}}^{\sigma} u(t)\right)=0, \quad 0<t<1, \\
u(0)=u^{\prime \prime}(0)=0, \quad u^{\prime}(1)={ }^{C} D_{0^{+}}^{\sigma} u(1),
\end{array}\right.
$$

where $f:[0,1] \times R \times R \rightarrow R$ is a given function, $2<q<3,0<\sigma<1$, and ${ }^{C} D_{0^{+}}^{q}$ represents the standard Caputo fractional derivative of order $q$.

Motivated by all the works above, this paper deals with the existence and uniqueness of solutions for the singular fractional boundary value problem with a fractional derivative condition,

$$
\left\{\begin{array}{l}
{ }^{C} D_{0^{+}}^{q} u(t)+f\left(t, u(t),{ }^{C} D_{0^{+}}^{\sigma} u(t)\right)=0, \quad 0<t<1, \\
u(0)=u^{\prime}(0)=0, \quad u^{\prime}(1)={ }^{C} D_{0^{+}}^{\sigma} u(1),
\end{array}\right.
$$

where $2<q<3,0<\sigma<1, f:(0,1] \times R \times R \rightarrow R$ is continuous, $f(t, x, y)$ may be singular at $t=0,{ }^{C} D_{0^{+}}^{q}$ is the standard Caputo derivative.

The paper is organized as follows. In Section 2, we shall introduce some definitions and lemmas to prove our main results. In Section 3, we establish some criteria for the existence for the boundary value problem (1.1) by using the Banach fixed point theorem and the Schauder fixed point theorem. Finally, we present two examples to illustrate our main results.

\section{Preliminaries and lemmas}

In this section, we present definitions and some fundamental facts from fractional calculus which can be found in [21].

Let $E=\left\{x: x \in C[0,1],{ }^{C} D_{0^{+}}^{\sigma} x \in C[0,1]\right\}, 0<\sigma<1$, endowed with the norm

$$
\|x\|=\max \left\{\max _{t \in[0,1]}|x(t)|, \max _{t \in[0,1]}\left|{ }^{C} D_{0^{+}}^{\sigma} x(t)\right|\right\},
$$

then $(E,\|\cdot\|)$ is a Banach space.

Definition 2.1 [21] If $g \in C[a, b]$ and $\alpha>0$, then the Riemann-Liouville fractional integral is defined as

$$
I_{a^{+}}^{\alpha} g(t)=\frac{1}{\Gamma(\alpha)} \int_{a}^{t}(t-s)^{\alpha-1} g(s) d s .
$$


Definition 2.2 [21] Let $\alpha>0, n=[\alpha]+1$. If $g \in A C^{n}([a, b])$, then the Caputo fractional derivative of order $\alpha$ defined by

$$
{ }^{C} D_{a^{+}}^{\alpha} g(t)=\frac{1}{\Gamma(n-\alpha)} \int_{a}^{t}(t-s)^{n-\alpha-1} g^{(n)}(s) d s
$$

exists almost everywhere on $[a, b]([\alpha]$ denotes the integer part of the real number $\alpha)$.

Lemma 2.1 [21] Let $\alpha, \beta>0$ and $n=[\alpha]+1$. Then the following relations hold:

$$
{ }^{C} D_{a^{+}}^{\alpha} t^{\beta-1}=\frac{\Gamma(\beta)}{\Gamma(\beta-\alpha)} t^{\beta-\alpha-1}, \quad \beta>n
$$

and

$$
{ }^{C} D_{a^{+}}^{\alpha} t^{k}=0, \quad k=0,1,2, \ldots, n-1 .
$$

Lemma 2.2 [21] For $\alpha>0, g(t) \in C[a, b]$, the homogeneous fractional differential equation ${ }^{C} D_{a^{+}}^{\alpha} g(t)=0$ has a solution

$$
g(t)=C_{1}+C_{2} t+C_{3} t^{2}+\cdots+C_{n} t^{n-1},
$$

where $C_{i}, i=1,2, \ldots, n$, and $n=[\alpha]+1$.

Lemma $2.3[21]$ Let $p, q \geq 0$, and $f \in L_{1}([a, b])$. Then

$$
\begin{aligned}
& I_{a^{+}}^{p} I_{a^{+}}^{q} f(t)=I_{a^{+}}^{p+q} f(t)=I_{a^{+}}^{q} I_{a^{+}}^{p} f(t), \\
& { }^{C} D_{a^{+}}^{q} I_{a^{+}}^{q} f(t)=f(t), \quad \forall t \in[a, b] .
\end{aligned}
$$

Lemma 2.4 [21] Let $\beta>\alpha>0$, and $f \in L_{1}([a, b])$. Then for all $t \in[a, b]$ we have

$$
{ }^{C} D_{a^{+}}^{\alpha} I_{a^{+}}^{\beta} f(t)=I_{a^{+}}^{\beta-\alpha} f(t) .
$$

Lemma 2.5 (Schauder fixed point theorem) Let $(E, d)$ be a complete metric space, let $U$ be a closed convex subset of $E$, and let $A: U \rightarrow U$ be a mapping such that the set $\{A u: u \in U\}$ is relatively compact in $E$. Then $A$ has at least one fixed point.

Lemma 2.6 For $y \in C[0,1]$ and $2<q<3,0<\sigma<1$, the unique solution of

$$
\left\{\begin{array}{l}
{ }^{C} D_{0^{+}}^{q} u(t)=y(t), \quad 0<t<1, \\
u(0)=u^{\prime}(0)=0, \quad u^{\prime}(1)={ }^{C} D_{0^{+}}^{\sigma} u(1),
\end{array}\right.
$$

is given by

$$
u(t)=\int_{0}^{1} G(t, s) y(s) d s
$$


where

$$
G(t, s)= \begin{cases}\frac{(t-s)^{q-1}}{\Gamma(2)}+\frac{\Gamma(3-\sigma) t^{2}(1-s)^{q-2}}{2 \Gamma(3-\sigma)-\Gamma(3)}\left(\frac{(1-s)^{1-\sigma}}{\Gamma(q-\sigma)}-\frac{1}{\Gamma(q-1)}\right), & 0 \leq s \leq t \leq 1, \\ \frac{\Gamma(3-\sigma) t^{2}(1-s)^{q-2}}{2 \Gamma(3-\sigma)-\Gamma(3)}\left(\frac{(1-s)^{1-\sigma}}{\Gamma(q-\sigma)}-\frac{1}{\Gamma(q-1)}\right), & 0 \leq t \leq s \leq 1 .\end{cases}
$$

Proof By Lemma 2.2, we get

$$
u(t)=I_{0^{+}}^{q} y(t)+C_{1}+C_{2} t+C_{3} t^{2},
$$

for some $C_{i} \in R, i=1,2,3$. So, we have

$$
u^{\prime}(t)=I_{0^{+}}^{q-1} y(t)+C_{2}+2 C_{3} t
$$

From the conditions $u(0)=u^{\prime}(0)=0$, we obtain $C_{1}=C_{2}=0$. Hence,

$$
{ }^{C} D_{0^{+}}^{\sigma} u(t)=I_{0^{+}}^{q-\sigma} y(t)+C_{3}{ }^{C} D_{0^{+}}^{\sigma} t^{2}=I_{0^{+}}^{q-\sigma} y(t)+C_{3} \frac{\Gamma(3)}{\Gamma(3-\sigma)} t^{2-\sigma} .
$$

The condition $u^{\prime}(1)={ }^{C} D_{0^{+}}^{\sigma} u(1)$ implies that

$$
C_{3}=\frac{\Gamma(3-\sigma)}{2 \Gamma(3-\sigma)-\Gamma(3)} \int_{0}^{1}\left(\frac{(1-s)^{q-\sigma-1}}{\Gamma(q-\sigma)}-\frac{(1-s)^{q-2}}{\Gamma(q-1)}\right) y(s) d s .
$$

Therefore, $u(t)$ can be written as

$$
\begin{aligned}
u(t) & =I_{0^{+}}^{q} y(t)+\frac{\Gamma(3-\sigma) t^{2}}{2 \Gamma(3-\sigma)-\Gamma(3)} \int_{0}^{1}\left(\frac{(1-s)^{q-\sigma-1}}{\Gamma(q-\sigma)}-\frac{(1-s)^{q-2}}{\Gamma(q-1)}\right) y(s) d s \\
& =\int_{0}^{1} G(t, s) y(s) d s,
\end{aligned}
$$

where $G(t, s)$ is defined by (2.1). The proof is complete.

\section{Existence and uniqueness results}

Define the operator $T: E \rightarrow E$ by

$$
T u(t)=\int_{0}^{1} G(t, s) f\left(s, u(s),{ }^{C} D_{0^{+}}^{\sigma} u(s)\right) d s, \quad \forall t \in[0,1] .
$$

Denote

$$
\begin{aligned}
& B_{1}=\frac{B(q, 1-\delta)}{\Gamma(q)}+\frac{\Gamma(3-\sigma)}{2 \Gamma(3-\sigma)-\Gamma(3)}\left(\frac{B(q-\sigma, 1-\delta)}{\Gamma(q-\sigma)}+\frac{B(q-1,1-\delta)}{\Gamma(q-1)}\right), \\
& B_{2}=\frac{B(q-\sigma, 1-\delta)}{\Gamma(q-\sigma)}+\frac{\Gamma(3)}{2 \Gamma(3-\sigma)-\Gamma(3)}\left(\frac{B(q-\sigma, 1-\delta)}{\Gamma(q-\sigma)}+\frac{B(q-1,1-\delta)}{\Gamma(q-1)}\right) .
\end{aligned}
$$

Lemma 3.1 Let $2<q<3,0<\delta<1, F:(0,1] \rightarrow R$ is continuous, and $\lim _{t \rightarrow 0^{+}} F(t)=\infty$. Suppose that $t^{\delta} F(t)$ is continuous on $[0,1]$. Then the function $u(t)=\int_{0}^{1} G(t, s) F(s) d s$ is continuous on $[0,1]$. 
Proof By the continuity of $t^{\delta} F(t)$ and $u(t)=\int_{0}^{1} G(t, s) s^{-\delta} s^{\delta} F(s) d s$. It is easy to know that $u(t)=0$. Now we separate the process into three cases.

Case 1 . For $t_{0}=0$ and $\forall t \in(0,1]$. Because of the continuity of $t^{\delta} F(t)$, there exists a constant $M>0$ such that $\left|t^{\delta} F(t)\right| \leq M, t \in[0,1]$, then

$$
\begin{aligned}
|u(t)-u(0)|= & \mid \int_{0}^{t} \frac{(t-s)^{q-1}}{\Gamma(q)} s^{-\delta} s^{\delta} F(s) d s+\frac{\Gamma(3-\sigma) t^{2}}{2 \Gamma(3-\sigma)-\Gamma(3)} \\
& \times \int_{0}^{1}\left(\frac{(1-s)^{q-\sigma-1}}{\Gamma(q-\sigma)}-\frac{(1-s)^{q-2}}{\Gamma(q-1)}\right) s^{-\delta} s^{\delta} F(s) d s \mid \\
\leq & M \int_{0}^{t} \frac{(t-s)^{q-1} s^{-\delta}}{\Gamma(q)} d s+\frac{\Gamma(3-\sigma) t^{2} M}{2 \Gamma(3-\sigma)-\Gamma(3)} \\
& \times \int_{0}^{1}\left(\frac{(1-s)^{q-\sigma-1} s^{-\delta}}{\Gamma(q-\sigma)}+\frac{(1-s)^{q-2} s^{-\delta}}{\Gamma(q-1)}\right) d s \\
= & \frac{M t^{q-\delta}}{\Gamma(q)} \int_{0}^{1}(1-w)^{q-1} w^{-\delta} d w+\frac{\Gamma(3-\sigma) t^{2} M}{2 \Gamma(3-\sigma)-\Gamma(3)} \\
& \times\left(\frac{B(q-\sigma, 1-\delta)}{\Gamma(q-\sigma)}+\frac{B(q-1,1-\delta)}{\Gamma(q-1)}\right) \\
= & \frac{M t^{q-\delta}}{\Gamma(q)} B(q, 1-\delta)+\frac{\Gamma(3-\sigma) t^{2} M}{2 \Gamma(3-\sigma)-\Gamma(3)} \\
& \times\left(\frac{B(q-\sigma, 1-\delta)}{\Gamma(q-\sigma)}+\frac{B(q-1,1-\delta)}{\Gamma(q-1)}\right) \\
\rightarrow & 0, \quad \text { as } t \rightarrow 0,
\end{aligned}
$$

where $B$ denotes the beta function.

Case 2. For $t_{0} \in(0,1)$ and $\forall t \in\left(t_{0}, 1\right]$, then

$$
\begin{aligned}
\left|u(t)-u\left(t_{0}\right)\right|= & \mid \int_{0}^{t} \frac{(t-s)^{q-1}}{\Gamma(q)} s^{-\delta} s^{\delta} F(s) d s-\int_{0}^{t_{0}} \frac{\left(t_{0}-s\right)^{q-1}}{\Gamma(q)} s^{-\delta} s^{\delta} F(s) d s \\
& +\frac{\Gamma(3-\sigma) t^{2}}{2 \Gamma(3-\sigma)-\Gamma(3)} \int_{0}^{1}\left(\frac{(1-s)^{q-\sigma-1}}{\Gamma(q-\sigma)}-\frac{(1-s)^{q-2}}{\Gamma(q-1)}\right) s^{-\delta} s^{\delta} F(s) d s \\
& -\frac{\Gamma(3-\sigma) t_{0}^{2}}{2 \Gamma(3-\sigma)-\Gamma(3)} \int_{0}^{1}\left(\frac{(1-s)^{q-\sigma-1}}{\Gamma(q-\sigma)}-\frac{(1-s)^{q-2}}{\Gamma(q-1)}\right) s^{-\delta} s^{\delta} F(s) d s \mid \\
\leq & \left|\int_{0}^{t_{0}} \frac{(t-s)^{q-1}-\left(t_{0}-s\right)^{q-1}}{\Gamma(q)} s^{-\delta} s^{\delta} F(s) d s+\int_{t_{0}}^{t} \frac{(t-s)^{q-1}}{\Gamma(q)} s^{-\delta} s^{\delta} F(s) d s\right| \\
& +\frac{\Gamma(3-\sigma)\left(t^{2}-t_{0}^{2}\right)}{2 \Gamma(3-\sigma)-\Gamma(3)} \int_{0}^{1}\left|\left(\frac{(1-s)^{q-\sigma-1}}{\Gamma(q-\sigma)}-\frac{(1-s)^{q-2}}{\Gamma(q-1)}\right) s^{-\delta} s^{\delta} F(s)\right| d s \\
\leq & \frac{M}{\Gamma(q)}\left(\int_{0}^{t_{0}}\left[(t-s)^{q-1}-\left(t_{0}-s\right)^{q-1} s^{-\delta} d s+\int_{t_{0}}^{t}(t-s)^{q-1} s^{-\delta} d s\right)\right. \\
& +\frac{\Gamma(3-\sigma)\left(t^{2}-t_{0}^{2}\right) M}{2 \Gamma(3-\sigma)-\Gamma(3)}\left(\frac{B(q-\sigma, 1-\delta)}{\Gamma(q-\sigma)}+\frac{B(q-1,1-\delta)}{\Gamma(q-1)}\right) \\
\leq & \frac{M\left(t^{q-\delta}-t_{0}^{q-\delta}\right) B(q, 1-\delta)}{\Gamma(q)}+\frac{\Gamma(3-\sigma)\left(t^{2}-t_{0}^{2}\right) M}{2 \Gamma(3-\sigma)-\Gamma(3)} \\
& \times\left(\frac{B(q-\sigma, 1-\delta)}{\Gamma(q-\sigma)}+\frac{B(q-1,1-\delta)}{\Gamma(q-1)}\right) \\
\rightarrow & 0, \quad \text { as } t \rightarrow t_{0} .
\end{aligned}
$$


Case 3. For $t_{0} \in(0,1]$ and $\forall t \in\left[0, t_{0}\right]$. The proof is similar to Case 2 , here we just leave it out. This completes the proof.

Lemma 3.2 Let $2<q<3,0<\delta<1, f:(0,1] \times R \times R \rightarrow R$ is continuous, and $\lim _{t \rightarrow 0^{+}} f(t$, $\cdot, \cdot)=\infty$. Suppose that $t^{\delta} f(t, \cdot, \cdot)$ is continuous on $[0,1] \times R \times R$. Then

$$
{ }^{C} D_{0^{+}}^{\sigma} T u(t)={ }^{C} D_{0^{+}}^{\sigma}\left(\int_{0}^{1} G(t, s) f\left(s, u(s),{ }^{C} D_{0^{+}}^{\sigma} u(s)\right) d s\right)
$$

is continuous on $[0,1]$.

Proof From $u \in E$ we obtain $u(t) \in C[0,1]$ and ${ }^{C} D_{0^{+}}^{\sigma} u(t) \in C[0,1]$. Hence, there exist two constants $L_{1}>0$ and $L_{2}>0$ such that $|u(t)| \leq L_{1},\left|{ }^{C} D_{0^{+}}^{\sigma} u(t)\right| \leq L_{2}$, for $t \in[0,1]$. Since $t^{\delta} f(t, \cdot, \cdot)$ is continuous on $[0,1] \times R \times R$, we have

$$
\begin{aligned}
& M_{0}=\max _{t \in[0,1]}\left|t^{\delta} f(t, u, v)\right|, \quad \text { for }-L_{1} \leq u \leq L_{1},-L_{2} \leq v \leq L_{2} \text {, } \\
& \left|{ }^{C} D_{0^{+}}^{\sigma} T u(t)\right|=\mid{ }^{C} D_{0^{+}}^{\sigma}\left(I_{0^{+}}^{q} f\left(t, u(t),{ }^{C} D_{0^{+}}^{\sigma} u(t)\right)\right)+\frac{\Gamma(3-\sigma) t^{2}}{2 \Gamma(3-\sigma)-\Gamma(3)} \\
& \times \int_{0}^{1}\left(\frac{(1-s)^{q-\sigma-1}}{\Gamma(q-\sigma)}-\frac{(1-s)^{q-2}}{\Gamma(q-1)}\right) f\left(s, u(s),{ }^{C} D_{0^{+}}^{\sigma} u(s)\right) d s \mid \\
& =\mid I_{0^{+}}^{q-\sigma} f\left(t, u(t),{ }^{C} D_{0^{+}}^{\sigma} u(t)\right)+\frac{\Gamma(3) t^{2-\sigma}}{2 \Gamma(3-\sigma)-\Gamma(3)} \\
& \times \int_{0}^{1}\left(\frac{(1-s)^{q-\sigma-1}}{\Gamma(q-\sigma)}-\frac{(1-s)^{q-2}}{\Gamma(q-1)}\right) f\left(s, u(s),{ }^{C} D_{0^{+}}^{\sigma} u(s)\right) d s \mid \\
& \leq M_{0} \int_{0}^{t} \frac{(t-s)^{q-\sigma-1} t^{-\sigma}}{\Gamma(q-\sigma)} d s+\frac{M_{0} \Gamma(3) t^{2-\sigma}}{2 \Gamma(3-\sigma)-\Gamma(3)} \\
& \times \int_{0}^{1}\left(\frac{(1-s)^{q-\sigma-1} s^{-\delta}}{\Gamma(q-\sigma)} d s+\frac{(1-s)^{q-2} s^{-\delta}}{\Gamma(q-1)}\right) d s \\
& =\frac{M_{0} t^{q-\sigma-\delta} B(q-\sigma, 1-\delta)}{\Gamma(q-\sigma)}+\frac{M_{0} \Gamma(3) t^{2-\sigma}}{2 \Gamma(3-\sigma)-\Gamma(3)} \\
& \times\left(\frac{B(q-\sigma, 1-\delta)}{\Gamma(q-\sigma)}+\frac{B(q-1,1-\delta)}{\Gamma(q-1)}\right) .
\end{aligned}
$$

Observing that $t^{q-\sigma-\delta}, t^{2-\sigma}$ are continuous on $[0,1]$, we can show ${ }^{C} D_{0^{+}}^{\sigma} T u(t)$ is continuous on $[0,1]$ by using the same method as in Lemma 3.1. The proof is completed.

Lemma 3.3 Let $2<q<3,0<\delta<1, f:(0,1] \times R \times R \rightarrow R$ is continuous, and $\lim _{t \rightarrow 0^{+}} f(t$, $\cdot, \cdot)=\infty$. Assume that $t^{\delta} f(t, \cdot, \cdot)$ is continuous on $[0,1] \times R \times R$. Then the operator $T: E \rightarrow E$ is completely continuous.

Proof For $\forall u \in E, T u(t)=\int_{0}^{1} G(t, s) f\left(s, u(s),{ }^{C} D_{0^{+}}^{\sigma} u(s)\right) d s$, by Lemma 3.1 and Lemma 3.2, we have $T: E \rightarrow E$. Now we separate the proof into three steps.

Step 1. Proof of $T: E \rightarrow E$ is continuous.

Let $u_{0} \in E$ and $\left\|u_{0}\right\|=C_{0}$. If $u \in E$ and $\left\|u-u_{0}\right\|<1$, then $\|u\|<1+C_{0}=C$. By the continuity of $t^{\delta} f\left(t, u(t),{ }^{C} D_{0^{+}}^{\sigma} u(t)\right)$, we know that $t^{\delta} f\left(t, u(t),{ }^{C} D_{0^{+}}^{\sigma} u(t)\right)$ is uniformly continuous 
on $[0,1] \times[-C, C] \times[-C, C]$. Thus for $\forall \varepsilon>0$, there exists $\eta>0(\eta<1)$, such that

$$
\left|t^{\delta} f\left(t, u(t),{ }^{C} D_{0^{+}}^{\sigma} u(t)\right)-t^{\delta} f\left(t, u_{0}(t),{ }^{C} D_{0^{+}}^{\sigma} u_{0}(t)\right)\right|<\varepsilon, \quad \text { for all } t \in[0,1],
$$

$u \in E$, with $\left\|u-u_{0}\right\|<\eta$.

It follows from (3.2) that

$$
\begin{aligned}
& \left|T u(t)-T u_{0}(t)\right| \\
& \quad \leq \int_{0}^{1}\left|G(t, s) s^{-\delta}\right|\left|s^{\delta} f\left(s, u(s),{ }^{C} D_{0^{+}}^{\sigma} u(s)\right)-s^{\delta} f\left(s, u_{0}(s),{ }^{C} D_{0^{+}}^{\sigma} u_{0}(s)\right)\right| d s \\
& \quad<\varepsilon \int_{0}^{1}\left|G(t, s) s^{-\delta}\right| d s \\
& \quad \leq \varepsilon\left(\int_{0}^{t} \frac{(t-s)^{q-1} s^{-\delta}}{\Gamma(q)} d s+\frac{\Gamma(3-\sigma) t^{2}}{2 \Gamma(3-\sigma)-\Gamma(3)} \int_{0}^{1}\left|\left(\frac{(1-s)^{q-\sigma-1}}{\Gamma(q-\sigma)}-\frac{(1-s)^{q-2}}{\Gamma(q-1)}\right) s^{-\delta}\right| d s\right) \\
& \quad \leq \varepsilon\left[\frac{t^{q-\delta} B(q, 1-\delta)}{\Gamma(q)}+\frac{\Gamma(3-\sigma) t^{2}}{2 \Gamma(3-\sigma)-\Gamma(3)}\left(\frac{B(q-\sigma, 1-\delta)}{\Gamma(q-\sigma)}+\frac{B(q-1,1-\delta)}{\Gamma(q-1)}\right)\right] \\
& \quad \leq \varepsilon B_{1} .
\end{aligned}
$$

On the other hand, by (3.1), we get

$$
\begin{aligned}
\left|{ }^{C} D_{0^{+}}^{\sigma} T u(t)-{ }^{C} D_{0^{+}}^{\sigma} T u_{0}(t)\right| & \left|{ }^{C} D_{0^{+}}^{\sigma}\left(T u(t)-T u_{0}(t)\right)\right| \\
= & \mid I_{0^{+}}^{q-\sigma}\left(f\left(t, u(t),{ }^{C} D_{0^{+}}^{\sigma} u(t)\right)-f\left(t, u_{0}(t),{ }^{C} D_{0^{+}}^{\sigma} u_{0}(t)\right)\right) \\
& +\frac{\Gamma(3) t^{2-\sigma}}{2 \Gamma(3-\sigma)-\Gamma(3)} \int_{0}^{1}\left(\frac{(1-s)^{q-\sigma-1}}{\Gamma(q-\sigma)}-\frac{(1-s)^{q-2}}{\Gamma(q-1)}\right) \\
& \times\left(f\left(s, u(s),{ }^{C} D_{0^{+}}^{\sigma} u(s)\right)-f\left(t, u_{0}(t),{ }^{C} D_{0^{+}}^{\sigma} u_{0}(t)\right)\right) d s \mid \\
\leq & \varepsilon\left[\frac{B(q-\sigma, 1-\delta)}{\Gamma(q-\sigma)}+\frac{\Gamma(3)}{2 \Gamma(3-\sigma)-\Gamma(3)}\left(\frac{B(q-\sigma, 1-\delta)}{\Gamma(q-\sigma)}+\frac{B(q-1,1-\delta)}{\Gamma(q-1)}\right)\right] \\
= & \varepsilon B_{2} .
\end{aligned}
$$

Therefore, $\left\|T u-T u_{0}\right\| \rightarrow 0$ as $\left\|u-u_{0}\right\| \rightarrow 0$, i.e., $T: E \rightarrow E$ is continuous.

Step 2. Let $\Omega \subset E$ be bounded, then there exists a positive constant $b$ such that $\|u\| \leq b$, $\forall u \in \Omega$. Since $t^{\delta} f\left(t, u(t),{ }^{C} D_{0^{+}}^{\sigma} u(t)\right)$ is continuous on $[0,1] \times[-b, b] \times[-b, b]$, we see that there exists a positive constant $L$ such that

$$
\left|t^{\delta} f\left(t, u(t),{ }^{C} D_{0^{+}}^{\sigma} u(t)\right)\right| \leq L, \quad \forall t \in[0,1], \forall u \in \Omega .
$$

Thus, by (3.3) and (3.4), we have

$$
\begin{aligned}
& |T u(t)| \leq \int_{0}^{1}\left|G(t, s) s^{-\delta}\right|\left|s^{\delta} f\left(s, u(s),{ }^{C} D_{0^{+}}^{\sigma} u(s)\right)\right| d s \leq L \int_{0}^{1}\left|G(t, s) s^{-\delta}\right| d s \leq L B_{1}, \\
& \left|{ }^{C} D_{0^{+}}^{\sigma} T u(t)\right|=\mid I_{0^{+}}^{q-\sigma} f\left(t, u(t),{ }^{C} D_{0^{+}}^{\sigma} u(t)\right)+\frac{\Gamma(3) t^{2-\sigma}}{2 \Gamma(3-\sigma)-\Gamma(3)}
\end{aligned}
$$




$$
\begin{aligned}
& \times \int_{0}^{1}\left(\frac{(1-s)^{q-\sigma-1}}{\Gamma(q-\sigma)}-\frac{(1-s)^{q-2}}{\Gamma(q-1)}\right) f\left(s, u(s),{ }^{C} D_{0^{+}}^{\sigma} u(s)\right) d s \mid \\
\leq & L B_{2} .
\end{aligned}
$$

So, $T(\Omega)$ is bounded.

Step 3. We will prove that $T(\Omega)$ is equicontinuous.

For all $t_{1}, t_{2} \in[0,1], t_{1}<t_{2}$ and $u \in \Omega$ we have

$$
\begin{aligned}
& \left|T u\left(t_{2}\right)-T u\left(t_{1}\right)\right| \\
& =\mid \frac{1}{\Gamma(q)} \int_{0}^{t_{1}}\left[\left(t_{2}-s\right)^{q-1}-\left(t_{1}-s\right)^{q-1}\right] s^{-\delta} s^{\delta} f\left(s, u(s),{ }^{C} D_{0^{+}}^{\sigma} u(s)\right) d s \\
& +\frac{1}{\Gamma(q)} \int_{t_{1}}^{t_{2}}\left(t_{2}-s\right)^{q-1} s^{-\delta} s^{\delta} f\left(s, u(s),{ }^{C} D_{0^{+}}^{\sigma} u(s)\right) d s \\
& +\frac{\Gamma(3-\sigma)\left(t_{2}^{2}-t_{1}^{2}\right)}{2 \Gamma(3-\sigma)-\Gamma(3)} \int_{0}^{1}\left(\frac{(1-s)^{q-\sigma-1}}{\Gamma(q-\sigma)}-\frac{(1-s)^{q-2}}{\Gamma(q-1)}\right) s^{-\delta} s^{\delta} f\left(s, u(s),{ }^{C} D_{0^{+}}^{\sigma} u(s)\right) d s \mid \\
& \leq \frac{L}{\Gamma(q)} \int_{0}^{t_{1}}\left[\left(t_{2}-s\right)^{q-1}-\left(t_{1}-s\right)^{q-1}\right] s^{-\delta} d s+\frac{L}{\Gamma(q)} \int_{t_{1}}^{t_{2}}\left(t_{2}-s\right)^{q-1} s^{\delta} d s \\
& +\frac{L \Gamma(3-\sigma)\left(t_{2}^{2}-t_{1}^{2}\right)}{2 \Gamma(3-\sigma)-\Gamma(3)}\left(\frac{B(q-\sigma, 1-\delta)}{\Gamma(q-\sigma)}+\frac{B(q-1,1-\delta)}{\Gamma(q-1)}\right) \\
& =\frac{L\left(t_{2}^{q-\delta}-t_{1}^{q-\delta}\right) B(q, 1-\delta)}{\Gamma(q)} \\
& +\frac{L \Gamma(3-\sigma)\left(t_{2}^{2}-t_{1}^{2}\right)}{2 \Gamma(3-\sigma)-\Gamma(3)}\left(\frac{B(q-\sigma, 1-\delta)}{\Gamma(q-\sigma)}+\frac{B(q-1,1-\delta)}{\Gamma(q-1)}\right), \\
& \left|{ }^{C} D_{0^{+}}^{\sigma} T u\left(t_{2}\right)-{ }^{C} D_{0^{+}}^{\sigma} T u\left(t_{1}\right)\right| \\
& =\mid \frac{1}{\Gamma(q-\sigma)} \int_{0}^{t_{1}}\left[\left(t_{2}-s\right)^{q-\sigma-1}-\left(t_{1}-s\right)^{q-\sigma-1}\right] s^{-\delta} s^{\delta} f\left(s, u(s),{ }^{C} D_{0^{+}}^{\sigma} u(s)\right) d s \\
& +\frac{1}{\Gamma(q-\sigma)} \int_{t_{1}}^{t_{2}}\left(t_{2}-s\right)^{q-\sigma-1} s^{-\delta} s^{\delta} f\left(s, u(s),{ }^{C} D_{0^{+}}^{\sigma} u(s)\right) d s \\
& +\frac{\Gamma(3)\left(t_{2}^{2-\sigma}-t_{1}^{2-\sigma}\right)}{2 \Gamma(3-\sigma)-\Gamma(3)} \int_{0}^{1}\left(\frac{(1-s)^{q-\sigma-1}}{\Gamma(q-\sigma)}-\frac{(1-s)^{q-2}}{\Gamma(q-1)}\right) s^{-\delta} s^{\delta} f\left(s, u(s),{ }^{C} D_{0^{+}}^{\sigma} u(s)\right) d s \mid \\
& \leq \frac{L}{\Gamma(q-\sigma)} \int_{0}^{t_{1}}\left[\left(t_{2}-s\right)^{q-\sigma-1}-\left(t_{1}-s\right)^{q-\sigma-1}\right] s^{-\delta} d s \\
& +\frac{L}{\Gamma(q-\sigma)} \int_{t_{1}}^{t_{2}}\left(t_{2}-s\right)^{q-\sigma-1} s^{\delta} d s \\
& +\frac{L \Gamma(3)\left(t_{2}^{2-\sigma}-t_{1}^{2-\sigma}\right)}{2 \Gamma(3-\sigma)-\Gamma(3)}\left(\frac{B(q-\sigma, 1-\delta)}{\Gamma(q-\sigma)}+\frac{B(q-1,1-\delta)}{\Gamma(q-1)}\right) \\
& =\frac{L\left(t_{2}^{q-\sigma-\delta}-t_{1}^{q-\sigma-\delta}\right) B(q-\sigma, 1-\delta)}{\Gamma(q-\sigma)} \\
& +\frac{L \Gamma(3)\left(t_{2}^{2-\sigma}-t_{1}^{2-\sigma}\right)}{2 \Gamma(3-\sigma)-\Gamma(3)}\left(\frac{B(q-\sigma, 1-\delta)}{\Gamma(q-\sigma)}+\frac{B(q-1,1-\delta)}{\Gamma(q-1)}\right) .
\end{aligned}
$$

As $t_{1} \rightarrow t_{2}$, the right-hand sides of the inequalities (3.5) and (3.6) tend to 0 , consequently $\left\|T u\left(t_{2}\right)-T u\left(t_{1}\right)\right\| \rightarrow 0$, i.e., $T(\Omega)$ is equicontinuous. 
By means of the Arzela-Ascoli theorem, we conclude that $T$ is completely continuous.

Now we are in the position to establish the main results.

Theorem 3.1 Assume that:

$\left(\mathrm{H}_{1}\right)$ There exist two constants $l>0$ and $0<\sigma<1$ such that

$$
t^{\delta}\left|f\left(t, x_{1}, z_{1}\right)-f\left(t, x_{2}, z_{2}\right)\right| \leq l\left(\left|x_{1}-x_{2}\right|+\left|z_{1}-z_{2}\right|\right),
$$

for each $t \in[0,1]$ and all $x_{1}, x_{2}, z_{1}, z_{2} \in R$.

$\left(\mathrm{H}_{2}\right) \theta=\max \left\{2 l B_{1}, 2 l B_{2}\right\}<1$.

Then the BVP (1.1) has a unique solution.

Proof We shall use the Banach fixed point theorem. For this, we need to verify that $T$ is a contraction. Let $u, v \in E$, then from $\left(\mathrm{H}_{1}\right)$ and (3.3)-(3.4) we obtain

$$
\begin{aligned}
&|T u(t)-T v(t)|\left|\int_{0}^{1} G(t, s)\left(f\left(s, u(s),{ }^{C} D_{0^{+}}^{\sigma} u(s)\right)-f\left(s, v(s),{ }^{C} D_{0^{+}}^{\sigma} v(s)\right)\right)\right| \\
& \leq \int_{0}^{1}\left|G(t, s) s^{-\delta}\right|\left|s^{\delta}\left(f\left(s, u(s),{ }^{C} D_{0^{+}}^{\sigma} u(s)\right)-f\left(s, v(s),{ }^{C} D_{0^{+}}^{\sigma} v(s)\right)\right)\right| d s \\
& \leq l \int_{0}^{1}\left|G(t, s) s^{-\delta}\right||u(s)-v(s)| d s+l \int_{0}^{1}\left|G(t, s) s^{-\delta}\right|\left|{ }^{C} D_{0^{+}}^{\sigma} u(s)-{ }^{C} D_{0^{+}}^{\sigma} v(s)\right| d s \\
& \leq 2 l\|u-v\| \int_{0}^{1}\left|G(t, s) s^{-\delta}\right| d s \\
& \leq 2 l B_{1}\|u-v\|, \\
&\left|{ }^{C} D_{0^{+}}^{\sigma} T u(t)-{ }^{C} D_{0^{+}}^{\sigma} T v(t)\right| \\
&=\mid \int_{0}^{t} \frac{(t-s)^{q-\sigma-1} s^{-\delta}}{\Gamma(q-\sigma)}\left[s^{\delta}\left(f\left(s, u(s),{ }^{C} D_{0^{+}}^{\sigma} u(s)\right)-f\left(s, v(s),{ }^{C} D_{0^{+}}^{\sigma} v(s)\right)\right)\right] d s \\
& \quad+\frac{\Gamma(3) t^{2-\sigma}}{2 \Gamma(3-\sigma)-\Gamma(3)} \int_{0}^{1}\left(\frac{(1-s)^{q-\sigma-1} s^{-\delta}}{\Gamma(q-\sigma)}-\frac{(1-s)^{q-2} s^{-\delta}}{\Gamma(q-1)}\right) \\
& \quad \times s^{\delta}\left(f\left(s, u(s),{ }^{C} D_{0^{+}}^{\sigma} u(s)\right)-f\left(s, v(s),{ }^{C} D_{0^{+}}^{\sigma} v(s)\right)\right) d s \mid \\
& \leq 2 l\|u-v\| B_{2} .
\end{aligned}
$$

Taking (3.7) and (3.8) into account, we acquire $\|T u-T v\| \leq \theta\|u-v\|$; then it is a contraction. As a consequence of the Banach fixed point theorem, we deduce that $T$ has a fixed point which is the unique solution of the BVP (1.1). The proof is complete.

Next, we will use the Schauder fixed point theorem to prove our result.

For the sake of convenience, we set

$$
L_{0}=\max _{t \in[0,1]} t^{\delta}\left|f\left(t, u(t),{ }^{C} D_{0^{+}}^{\sigma} u(t)\right)\right|, \quad r=\max \left\{L_{0} B_{1}, L_{0} B_{2}\right\} .
$$


Theorem 3.2 Assume that $2<q<3,0<\delta<1, f:(0,1] \times R \times R \rightarrow R$ is continuous, and $\lim _{t \rightarrow 0^{+}} f(t, \cdot, \cdot)=\infty, t^{\delta} f(t, \cdot, \cdot)$ is continuous on $[0,1] \times R \times R$. Then the BVP (1.1) has a solution.

Proof Let $U=\{u: u \in E:\|u\| \leq r\}$. First, we prove that $T: U \rightarrow U$.

In fact, for each $t \in[0,1]$, we have

$$
\begin{aligned}
|T u(t)| \leq & \frac{1}{\Gamma(q)} \int_{0}^{t}(t-s)^{q-1} s^{-\delta}\left|s^{-\delta} f\left(s, u(s),{ }^{C} D_{0^{+}}^{\sigma} u(s)\right)\right| d s \\
& +\frac{\Gamma(3-\sigma) t^{2}}{2 \Gamma(3-\sigma)-\Gamma(3)} \int_{0}^{1}\left|\left(\frac{(1-s)^{q-\sigma-1} s^{-\delta}}{\Gamma(q-\sigma)}-\frac{(1-s)^{q-2} s^{-\delta}}{\Gamma(q-1)}\right)\right| \\
& \times\left|s^{\delta} f\left(s, u(s),{ }^{C} D_{0^{+}}^{\sigma} u(s)\right)\right| d s \\
\leq & L_{0} B_{1}, \\
\left|{ }^{C} D_{0^{+}}^{\sigma} T u(t)\right| \leq & \frac{1}{\Gamma(q-\sigma)} \int_{0}^{t}(t-s)^{q-\sigma-1} s^{-\delta}\left|s^{-\delta} f\left(s, u(s),{ }^{C} D_{0^{+}}^{\sigma} u(s)\right)\right| d s \\
& +\frac{\Gamma(3) t^{2-\sigma}}{2 \Gamma(3-\sigma)-\Gamma(3)} \int_{0}^{1}\left|\left(\frac{(1-s)^{q-\sigma-1} s^{-\delta}}{\Gamma(q-\sigma)}-\frac{(1-s)^{q-2} s^{-\delta}}{\Gamma(q-1)}\right)\right| \\
& \times\left|s^{\delta} f\left(s, u(s),{ }^{C} D_{0^{+}}^{\sigma} u(s)\right)\right| d s \\
\leq & L_{0} B_{2} .
\end{aligned}
$$

Hence, we can conclude that

$$
\|T u\|=\max \left\{\max _{t \in[0,1]}|T u(t)|, \max _{t \in[0,1]}\left|{ }^{C} D_{0^{+}}^{\sigma} T u(t)\right|\right\} \leq r .
$$

From Lemma 3.1 and Lemma 3.2, we know that $T u(t) \in C[0,1],{ }^{C} D_{0^{+}}^{\sigma} T u(t) \in C[0,1]$. Consequently, $T: U \rightarrow U$. From Lemma 3.3, we find that $T: U \rightarrow U$ is completely continuous. By Lemma 2.5, we deduce that the problem (1.1) has a solution. This completes the proof.

\section{Examples}

We illustrate our work with two examples.

Example 4.1 Consider the following fractional boundary value problem:

$$
\left\{\begin{array}{l}
{ }^{C} D_{0^{+}}^{\frac{14}{5}} u=t^{-\frac{1}{2}}\left(0.029 u+0.028^{C} D_{0^{+}}^{\frac{4}{5}} u+\cos t\right), \quad 0<t<1, \\
u(0)=u^{\prime}(0)=0, \quad u^{\prime}(1)={ }^{C} D_{0^{+}}^{\frac{4}{5}} u(1) .
\end{array}\right.
$$

We have

$$
f(t, x, y)=0.029 x+0.028 y+\cos t, \quad 2<q=\frac{14}{5}<3, \quad \sigma=\frac{4}{5}<1
$$

and

$$
t^{\frac{1}{2}}\left|f\left(t, x_{1}, z_{1}\right)-f\left(t, x_{2}, z_{2}\right)\right|<0.029\left(\left|x_{1}-x_{2}\right|+\left|z_{1}-z_{2}\right|\right),
$$


where $\delta=\frac{1}{2}<1, l=0.029$. Simple calculus gives

$$
\begin{aligned}
B_{1} & =\frac{B(q, 1-\delta)}{\Gamma(q)}+\frac{\Gamma(3-\sigma)}{2 \Gamma(3-\sigma)-\Gamma(3)}\left(\frac{B(q-\sigma, 1-\delta)}{\Gamma(q-\sigma)}+\frac{B(q-1,1-\delta)}{\Gamma(q-1)}\right) \\
& =16.0968 \\
B_{2} & =\frac{B(q-\sigma, 1-\delta)}{\Gamma(q-\sigma)}+\frac{\Gamma(3)}{2 \Gamma(3-\sigma)-\Gamma(3)}\left(\frac{B(q-\sigma, 1-\delta)}{\Gamma(q-\sigma)}+\frac{B(q-1,1-\delta)}{\Gamma(q-1)}\right) \\
& =16.7697 .
\end{aligned}
$$

So, $\theta=\max \left\{2 l B_{1}, 2 l B_{2}\right\}=0.9726<1$, then by Theorem 3.1 , the problem $(4.1)$ has a unique solution.

Remark 4.1 If $l>0.03$, then $\theta>1$. However, by Theorem 3.2, the problem (4.1) still has a solution.

Example 4.2 Let us consider the fractional boundary value problem

$$
\left\{\begin{array}{l}
{ }^{C} D_{0^{+}}^{\frac{14}{5}} u=t^{-\frac{1}{2}}\left(e^{-t} u^{2}+\left({ }^{C} D_{0^{+}}^{\frac{4}{5}} u\right)^{3}+(1-t)^{2}\right), \quad 0<t<1, \\
u(0)=u^{\prime}(0)=0, \quad u^{\prime}(1)={ }^{C} D_{0^{+}}^{\frac{4}{5}} u(1) .
\end{array}\right.
$$

Let $\sigma=\frac{1}{2}$, then all conditions in Theorem 3.2 are satisfied. Then the problem (4.2) has a solution.

\section{Competing interests \\ The author declares to have no competing interests.}

\section{Author's contributions}

The author read and approved the final manuscript.

\section{Acknowledgements}

The author is very grateful to the referees for their very helpful comments and suggestions, which greatly improved the presentation of this paper.

Received: 9 September 2014 Accepted: 12 November 2014 Published: 25 Nov 2014

\section{References}

1. Oldham, KB, Spanier, J: The Fractional Calculus. Academic Press, New York (1974)

2. Kilbas, AA, Srivastava, HM, Trujillo, JJ: Theory and Applications of Fractional Differential Equations. Elsevier, Amsterdam (2006)

3. Zhang, XG, Liu, LS, Wu, YH, Lu, Y: The iterative solutions of nonlinear fractional differential equations. Appl. Math. Comput. 219, 4680-4691 (2013)

4. Zhang, XG, Liu, LS, Wu, YH: The uniqueness of positive solutions for a singular fractional differential system involving derivatives. Commun. Nonlinear Sci. Numer. Simul. 18, 1400-1409 (2013)

5. Nieto, JJ, Pimentel, J: Positive solutions of a fractional thermostat model. Bound. Value Probl. 2013, 5 (2013)

6. Zhang, XG, Liu, LS, Wu, YH: The eigenvalue problem for a singular higher fractional differential equation involving fractional derivatives. Appl. Math. Comput. 218, 8526-8536 (2012)

7. Zhang, XG, Liu, LS, Wu, YH: Existence results for multiple positive solutions of nonlinear higher order perturbed fractional differential equations with derivatives. Appl. Math. Comput. 219, 1420-1433 (2012)

8. Zhang, XG, Liu, LS, Wiwatanapataphee, B, Wu, YH: Positive solutions of eigenvalue problems for a class of fractional differential equations with derivatives. Abstr. Appl. Anal. 2012, Article ID 512127 (2012)

9. Zhang, XG, Liu, LS, Wu, YH: Multiple positive solutions of a singular fractional differential equation with negatively perturbed term. Math. Comput. Model. 55, 1263-1274 (2012)

10. Zhao, Y, Sun, S, Han, Z, Li, Q: Theory of fractional hybrid differential equations. Comput. Math. Appl. 62, 1312-1324 (2011)

11. Feng, W, Sun, S, Han, Z, Zhao, Y: Existence of solutions for a singular system of nonlinear fractional differential equations. Comput. Math. Appl. 62, 1370-1378 (2011) 
12. Bai, Z, Lü, H: Positive solutions for boundary value problem of nonlinear fractional differential equation. J. Math. Anal. Appl. 311, 495-505 (2005)

13. Zhang, S: Positive solutions for boundary-value problems of nonlinear fractional differential equations. Electron. J. Differ. Equ. 2006, 36 (2006)

14. Sun, S, Zhao, Y, Han, Z, Li, Y: The existence of solutions for boundary value problem of fractional hybrid differential equations. Commun. Nonlinear Sci. Numer. Simul. 17, 4961-4967 (2012)

15. Sun, $\mathrm{S}, \mathrm{Zhao}, \mathrm{Y}, \mathrm{Han}, \mathrm{Z}, \mathrm{Xu}, \mathrm{M}$ : Uniqueness of positive solutions for boundary value problems of singular fractional differential equation. Inverse Probl. Sci. Eng. 20, 299-309 (2012)

16. El-Shahed, M, Nieto, JJ: Nontrivial solutions for a nonlinear multi-point boundary value problem of fractional order. Comput. Math. Appl. 59, 3438-3443 (2010)

17. Rehman, MU, Khan, RA: Existence and uniqueness of solutions for boundary value problems for fractional differential equation. Appl. Math. Lett. 23, 1038-1044 (2010)

18. Agarwal, RP, O'Regan, D, Stanek, S: Positive solutions for Dirichlet problems of singular nonlinear fractional differential equation. J. Math. Anal. Appl. 371, 57-68 (2010)

19. Yan, R, Sun, $S, L u, H$, Zhao, Y: Existence of solutions for fractional differential equations with integral boundary conditions. Adv. Differ. Equ. 2014, 25 (2014)

20. Guezane-Lakoud, A, Bensebaa, S: Solvability of a fractional boundary value problem with fractional derivative condition. Arab. J. Math. 3, 39-48 (2014)

21. Kilbas, AA, Srivastava, HM, Trujillo, Jj: Theory and Applications of Fractional Differential Equations. North-Holland Mathematics Studies, vol. 204. Elsevier, Amsterdam (2006)

10.1186/1687-1847-2014-292

Cite this article as: Li: Existence of solutions for nonlinear singular fractional differential equations with fractional

derivative condition. Advances in Difference Equations 2014, 2014:292

\section{Submit your manuscript to a SpringerOpen ${ }^{\circ}$ journal and benefit from:}

- Convenient online submission

- Rigorous peer review

- Immediate publication on acceptance

Open access: articles freely available online

- High visibility within the field

- Retaining the copyright to your article 